\title{
Obliteración de derivación gastrorrenal secundaria a terapia de várice gástrica fúndica con cianoacrilato guiada por ultrasonido endoscópico
}

\author{
Enrique Murcio-Pérez*, Raúl Zamarripa-Mottú, Julio C. Pintor-Belmontes, Gerardo Blanco-Velasco, \\ Omar M. Solórzano-Pineda y Oscar V. Hernández-Mondragón
}

Departamento de Endoscopia, Hospital de Especialidades, Centro Médico Nacional Siglo XXI, Instituto Mexicano del Seguro Social, Ciudad de México, México

\section{Resumen}

Las várices gástricas están presentes en alrededor del 20\% de los pacientes con cirrosis. El tratamiento endoscópico de la hemorragia secundaria a várices gástricas de afección fúndica aislada (IGV-1) es la inyección intravariceal de cianoacrilato. Entre las alternativas de tratamiento está la terapia vascular guiada por ultrasonido endoscópico (TV-USE). Se presenta un caso de hemorragia digestiva secundaria a IGV-1 con falla a tratamiento endoscópico convencional con cianoacrilato y respuesta satisfactoria a TV-USE, que favoreció oclusión de la várice gástrica y de derivación gastrorrenal.

Palabras clave: Várices gástricas. Endosonografía. Cianoacrilato.

\section{Endoscopic ultrasound guided cyanoacrylate gastro-renal shunt obliteration during fundic gastric varix therapy}

\begin{abstract}
Gastric varices are present in about $20 \%$ of cirrhotic patients. Intravariceal cyanoacrylate injection is the first line endoscopic treatment for isolated gastric fundal varices (IGV-1). Endoscopic ultrasound (EUS) guided therapy is a useful alternative. We present a case of EUS guided cyanoacrylate therapy of IGV-1, that also promote gastro-renal shunt obliteration.
\end{abstract}

Key words: Esophageal and gastric varices. Endosonography. Cyanoacrylates.

\section{Introducción}

Las várices gástricas están presentes en alrededor del $20 \%$ de los pacientes con cirrosis y tienen una tasa anual de hemorragia del $16 \%$ por año ${ }^{1}$.

El tratamiento endoscópico de la hemorragia por várices gástricas de afección fúndica aislada (IGV-1) es la inyección intravariceal de cianoacrilato ${ }^{2}$. Entre las alternativas de tratamiento está la terapia vascular guiada por ultrasonido endoscópico (TV-USE).

Se presenta un caso de hemorragia digestiva secundaria a IGV-1 con falla a tratamiento endoscópico convencional con cianoacrilato y respuesta satisfactoria a TV-USE, que favoreció oclusión de la várice gástrica y de derivación gastrorrenal. 


\section{Caso clínico}

Hombre de 37 años con abuso de alcohol que presentó hematemesis y melena. Se documentó IGV-1 y se inyectaron $2 \mathrm{ml}$ de cianoacrilato mediante videoendoscopio. Recibió manejo complementario con octreótida y antibiótico. A pesar de ello, persistió con melena y necesidad de hemotransfusión de 8 paquetes globulares. Siete días después presentó hematemesis asociada a hipotensión, por lo que se realizó una segunda endoscopia, que no evidenció el sitio de hemorragia debido a coágulos que impedían visión. Se colocó sonda de balones y el paciente se envió a hospital de tercer nivel.

Se retiró sonda de balones, encontrando coágulos que impidieron visualización endoscópica del fondo gástrico. Se introdujo ecoendoscopio lineal, logrando identificar zona anecoica, dilatada y tortuosa con flujo Doppler positivo compatible con várice fúndica (Fig. 1). Se identificó vaso perforante y se inyectaron $2 \mathrm{ml}$ de cianoacrilato en dilución con lipiodol 1:1 con aguja calibre $22 \mathrm{G}$. Se verificó ausencia de señal Doppler postinyección.

Posteriormente se realizó tomografía contrastada para determinar la permeabilidad del sistema porta y en búsqueda de derivaciones vasculares espontáneas, evidenciando ocupación de cianoacrilato en várice gástrica y oclusión completa de derivación gastrorrenal (Fig. $2 \mathrm{~A}$ y B). En ultrasonido endoscópico de control a las seis semanas persistía la ausencia de señal Doppler en várice gástrica.

\section{Discusión}

Las IGV-1 pueden asociarse a trombosis del sistema porta y a derivaciones vasculares espontáneas (gastrorrenal y gastrocava) ${ }^{3}$. En el presente caso, la oclusión con cianoacrilato se extendió favorablemente hacia la derivación gastrorrenal. Dicho evento consideramos que fue secundario a una inyección cercana al vaso perforante originado en la derivación vascular que otorgaba el flujo sanguíneo a la IGV-1. Hasta nuestro conocimiento, este es el primer caso que reporta obliteración completa de una derivación vascular mediante TV-USE.

La TV-USE tiene varias ventajas técnicas: visualización Doppler a pesar de la obstrucción visual por alimento o coágulos, identificación del vaso perforante y verificación en tiempo real de la ausencia de flujo postratamiento ${ }^{4}$. Una ventaja clínica de la TV-USE es menor recurrencia de hemorragia que la inyección convencional de cianoacrilato por endoscopia. La recurrencia de hemorragia de la TV-USE es menor en comparación con la terapia convencional ( 18.5 vs. $44.7 \% ; p=0.0053$ )

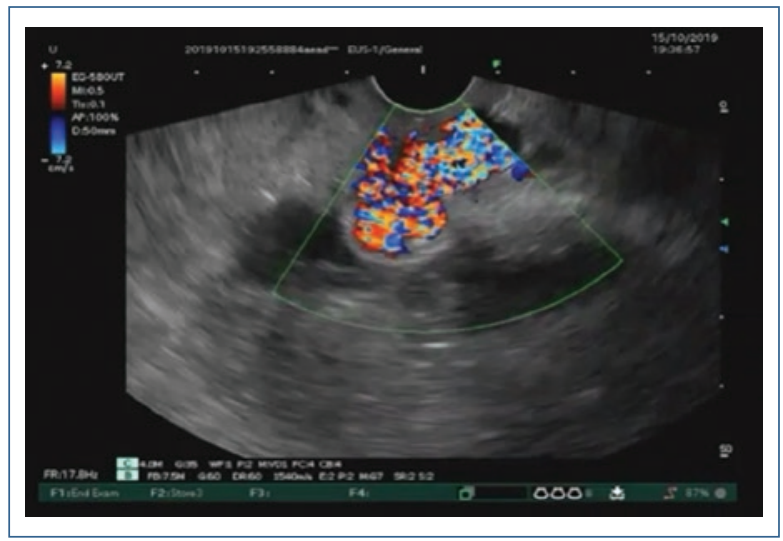

Figura 1. Visión endosonográfica de várice fúndica con señal Doppler positiva.

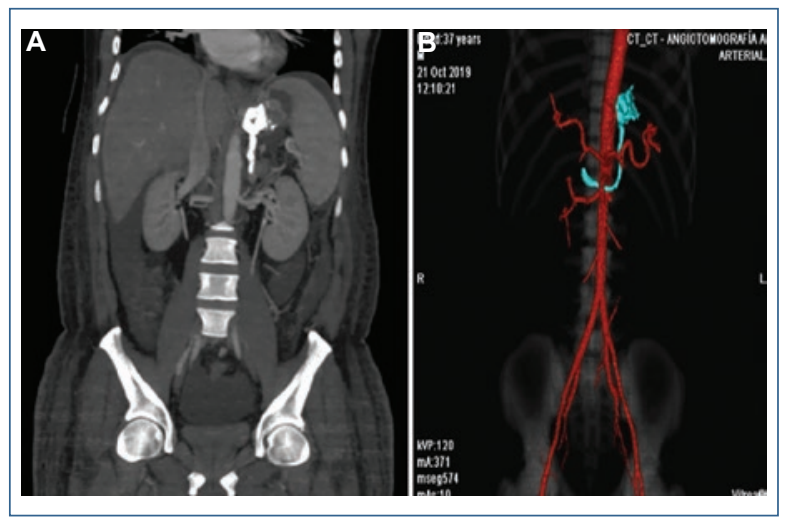

Figura 2. Tomografía abdominal en corte coronal que muestra distribución de cianoacrilato inyectado por ultrasonido endoscópico en várice fúndica con extensión a derivación vascular (A) y reconstrucción que muestra oclusión de várice fúndica y de derivación gastrorrenal (B).

disminuyendo un $72 \%$ dicho riesgo (odds ratio: 0,28 ; intervalo de confianza 95\%: 0,12-0,69) ${ }^{5}$.

\section{Conclusión}

La TV-USE es una alternativa eficaz para el tratamiento de las IGV-1 y tiene menor tasa de recurrencia de hemorragia en comparación con la terapia convencional con cianoacrilato.

\section{Financiamiento}

La presente investigación no ha recibido ninguna beca específica de agencias de los sectores público, comercial o sin ánimo de lucro. 


\section{Conflicto de intereses}

Los autores declaran no tener conflicto de intereses.

\section{Responsabilidades éticas}

Protección de personas y animales. Los autores declaran que para esta investigación no se han realizado experimentos en seres humanos ni en animales.

Confidencialidad de los datos. Los autores declaran que han seguido los protocolos de su centro de trabajo sobre la publicación de datos de pacientes.

Derecho a la privacidad y consentimiento informado. Los autores han obtenido el consentimiento informado de los pacientes y/o sujetos referidos en el artículo. Este documento obra en poder del autor de correspondencia.

\section{Bibliografía}

1. Sarin SK, Lahoti D, Saxena SP, Murthy NS, Makwana UK. Prevalence, classification and natural history of gastric varices: a long-term follow up study in 568 portal hypertension patients. Hepatology. 1992;16:1343-9.

2. Rios-Castellanos E, Seron P, Gisbert JP, Bonfill Cosp X. Endoscopic injection of cyanoacrylate glue versus other endoscopic procedures for acute bleeding gastric varices in people with portal hypertension. Cochrane Database Syst Rev. 2015 May 12;(5):CD010180.

3. García-Tsao G, Abraldes JG, Berzigotti A, Bosch J. Portal hypertensive bleeding in cirrhosis: Risk stratification, diagnosis and management: 2016 practice guidance by the American Association for the study of liver diseases. Hepatology. 2017;65(1):310-35.

4. Fujii-Lau LL, Law R, Wong Kee Song LM, Levy MJ. Novel techniques for gastric variceal obliteration. Dig Endosc. 2015;27:189-96.

5. Lee YT, Chan FK, Ng EK, Leung VK, Law KB, Yung MY, et al. EUS-guided injection of cyanoacrylate for bleeding gastric varices. Gastrointest Endosc. 2000;52(2):168-74. 